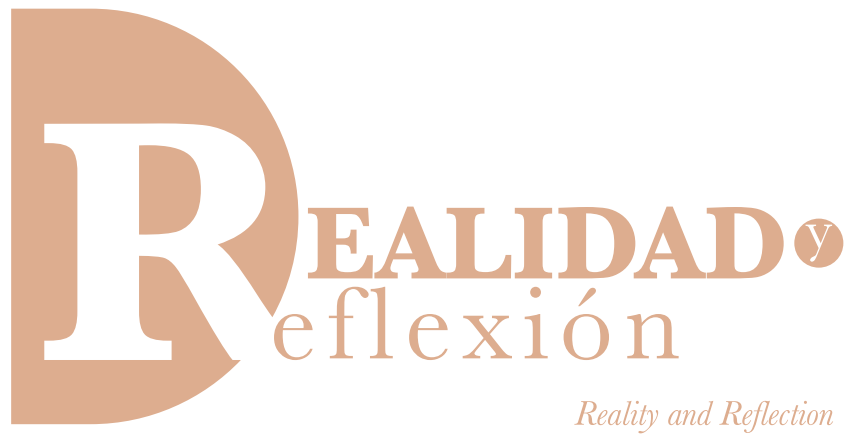

ISSN 1992-6510

e-ISSN 2520-9299

Año 17, N 46, San Salvador, El Salvador, Centroamérica. Revista Semestral Julio-Diciembre 2017

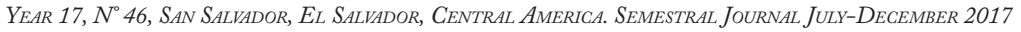

\title{
La posibilidad de una ética en realidades alternas. Un ejercicio académico
}

\section{The possibility of an ethic in alternate realities. An academic activity}

\author{
Dr. José Carlos Vázquez Parra ${ }^{1}$ \\ Escuela de Humanidades y Educación, Tecnológico de Monterrey en Guadalajara \\ Licenciado en Derecho, Licenciado en Psicología y Maestro en Educación por la Universidad del Valle de Atemajac. \\ Doctor en Estudios Humanísticos por el Tecnológico de Monterrey en Monterrey. \\ jcvazquezp@itesm.mx
}

Mtro. Emerson Michael Peña Camacho Escuela de Humanidades y Educación, Tecnológico de Monterrey en Guadalajara Licenciado en Filosofía y Maestro en Estudios Filosóficos por la Universidad de Guadalajara, Doctorando en Filosofía por el Instituto de investigaciones filosóficas IIF "Luis Villoro" de la Universidad Michoacana de San Nicolás de Hidalgo emerson@itesm.mx

Fecha de recepción: 19 de junio de 2017 Fecha aprobación: 5 de diciembre de 2017 pág. $22-33$ DOI: http://dx.doi.org/10.5377/ryr.v0i46.5505

\section{RESUMEN}

No cabe duda que el trabajo de formar nuevos profesionistas es una labor cada vez más compleja, sobre todo, cuando lo que se busca enseñar es sobre la ética del comportamiento y las decisiones que se puedan tomar en un futuro. El presente artículo muestra los resultados de un ejercicio académico realizado con alumnos de pregrado de una universidad privada mexicana, que, aunque era una actividad con el objetivo de desarrollar el juicio moral de sus participantes, terminó dando pauta a una reflexión nutrida e interesante sobre la posibilidad de la ética en realidades alternas. Aunque puede llegar a cuestionarse el cumplimiento de su hipótesis central, las conclusiones alcanzadas por los estudiantes son dignas de ser compartidas en este artículo.

Palabras clave: Juicio Moral, Black Mirror, Rick y Morty, Mundos alternos.

\section{ABSTRACT}

There is no doubt that the work of forming new professionals is an increasingly complex task, especially when what is sought to be taught is about behavioral ethics and decisions that may be taken in the future. This article shows the results of an academic exercise carried out with undergraduate students from a private Mexican university, which although it was an activity with the objective of developing the moral judgment of its participants, ended up giving a nuanced and interesting reflection on the possibility of ethics in alternate realities. Although the fulfillment of their central hypothesis may be questioned, the conclusions reached by the students are worthy of being shared in this article.

Keywords: Moral Judgment, Black Mirror, Rick and Morty, Alternate Worlds. 


\section{Introducción}

Uno de los retos que se tienen como profesor de ética en la época contemporánea, es poder contar con elementos de análisis óptimos para que los jóvenes puedan desarrollar una autonomía moral ante los problemas que viven, ya que no se puede negar que se vive en una realidad compleja, la cual requiere de ciudadanos íntegros y éticamente preparados.

Un medio por el cual los profesores acceden a estos procesos académicos es por medio de las tecnologías de la información y la comunicación (TIC), las cuales, abren un espacio claramente innovador para el desarrollo de competencias en los estudiantes.

Las TIC son herramientas que tienen como objetivo mejorar la comunicación entre las personas, incluyendo todos los medios audiovisuales (CENDI, 2012). Según sus características, las TIC pueden priorizar la transmisión o el procesamiento de la información, lo cual permite que éstas puedan relacionarse con procesos de interacción con los usuarios, incluidos los procesos educativos (Fernandez, 2014).

Algo innovador a lo que se tiene acceso con el uso de las TIC, en procesos educativos, es que permiten aproximaciones a realidades mucho más amplias que las que constituyen el entorno del estudiante, incluyendo incluso a aquellas creadas especialmente para detonar la reflexión y el análisis (Carrillo, 2005).

Sin embargo, aunque esto puede llegar a ser altamente benéfico para el desarrollo de competencias, también puede dar pauta a un conflicto, ya que, ¿Es posible y conveniente hacer reflexiones ético-morales en una realidad alternativa aunque ésta sea generada de manera ficticia por los medios de comunicación?, ¿Acaso se puede hacer un juicio, sustentado en valores de nuestra realidad, sobre dilemas que se enmarcan en una realidad virtual o mediática recreada?, ¿Es lo mismo hablar de realidades alternativas paralelas que realidades alternativas jerarquizadas?, ¿Acaso no se está ante el riesgo de una inconmensurabilidad por estar comparando realidades diferentes?

El presente artículo busca hacer una reflexión sobre la posibilidad de llevar a cabo este tipo de prácticas educativas, usando como ejemplo, un ejercicio académico realizado en un grupo de estudiantes de pregrado de una institución privada mexicana. Este análisis, se ha realizado considerando el uso de TIC y realidades mediáticas, usando como plataforma de referencia, el análisis de dilemas éticos en la serie Black Mirror y las aportaciones sobre realidades alternativas de la serie de dibujos animados Rick y Morty. La intención educativa, es desarrollar en los alumnos la competencia ética, haciéndolos conscientes de la posibilidad o imposibilidad de realizar juicios sobre las realidades, propias y alternativas, cuando éstas simbolizan o no una totalidad.

\section{Marco teórico}

Cuando se habla de realidades, es común que se caiga en una reflexión que puede pecar de simple ya que la comprensión de "realidad" puede centrarse exclusivamente en lo que comprendemos por "lo real", enfocándonos en "la totalidad de lo existente". Desde esta perspectiva, las "otras realidades" realmente no se 
conciben como tales, ya que al final de cuentas se conectan a nuestra realidad y, por ende, son parte de la misma (Harman, 2010).

Sin embargo, la cuestión puede llegar a ser mucho más compleja cuando se entiende que las "otras realidades" contienen características propias que son ajenas a las estructuras de la "realidad material", entre las cuales puede incluirse una normatividad moral alternativa.

Esta situación, parece cuestionar el sentido de universalidad como totalidad, que en ocasiones se presume cuando se hacen planteamientos sobre la realidad. Lyotard (1991) plantea que este sentido de la universalidad puede ser cuestionado, considerando que lo universal responde más a la idea de una objetividad en el conocimiento, o bien, a la legitimidad de las normas en vigor, no tanto así a un todo que no da lugar a duda o incertidumbre.

Esto es semejante a lo que Bauman (1996) propone cuando señala las diferencias entre la modernidad y la posmodernidad en su Teoría Sociológica de la Posmodernidad, en la cual se señala que la idea de un universalismo como unidad, claridad y certeza, llega a modificarse en la posmodernidad, en la que la realidad se enmarca en situaciones globalizantes, de amplia diversidad y de inevitable incertidumbre. La postmodernidad se identifica con el pluralismo institucionalizado, la diversidad, la casualidad y la ambivalencia, dando pauta a la posibilidad de espacios alternos, no pudiendo existir un universalismo como totalidad.

Cuando se llevan cursos de ética, enmarcados por teóricos posmodernos, los profesores enfrentan claros retos al tratar de hacer que los estudiantes alcancen a comprender estas precisiones de lo que se entiende por universalismo, lo cual suele contrastarse con la normatividad moral que pertenece a un grupo, lugar o tiempo determinado. La comprensión de la universalidad como parte de una realidad, $y$ no como una totalidad, se vuelve en un punto de análisis que incluso muchos académicos no consiguen precisar, y que por ende, los lleva a negar la posibilidad de hacer análisis sobre la existencia de realidades alternativas.

Una forma de aclarar las diferencias entre una realidad universal como totalidad, de una realidad alternativa y derivada, es a partir de algunos ejemplos que dan diversas series televisivas, como la que se usó de caso de estudio en el presente artículo.

Primero, se quiere presentar el ejemplo de dos capítulos de la serie animada Rick and Morty. De manera específica se hace uso de los episodios \#10, "Rickcuentros en la fase Rick" y \#17, “Los Ricks deben estar locos”. De manera general, en esta serie, Rick ha logrado crear un artefacto capaz de entrar a distintas realidades o posibilidades de realidad, a las que arrastra a su nieto Morty (Salcedo, 2013). De manera concreta en estos capítulos, la serie muestra la posibilidad de realidades alternas, aunque en cada uno de los episodios se concibe que lo alterno puede gestarse de formas distintas (Evans, 2015).

En el episodio 10, un grupo de Ricks de realidades alternativas paralelas juzgan a Rick por crímenes realizados contra otros Ricks. Aunque esto resulta confuso, no lo es cuando se comprende que en cada realidad hay un Rick 
y un correspondiente Morty (R1-R2-R3, etc.). De esta forma, la posibilidad de viajar entre realidades también puede llevar al encuentro inevitable consigo mismo.

A diferencia de esto, en el capítulo 17, Rick tiene una batería que en realidad es un microuniverso que ha sido creado por él mismo y que tiene como objetivo la generación de energía, llevando a que sus habitantes tengan como único propósito de vida alimentar este acumulador. Sin embargo, lo que no había precisado Rick, es que esta realidad que vive en su batería ha creado una segunda batería bajo el mismo principio, con el fin de liberar a sus ciudadanos de las pesadas tareas de producir energía. Sin alargar la explicación, la cual se desarrolla cuestionando la posibilidad o no de esclavitud de los pobladores de los microuniversos, esto llega hasta un cuarto nivel, en el que uno de los científicos de dicha realidad termina suicidándose, al descubrir por boca de Morty, que su mundo es una realidad creada con el único objetivo de ser usado como batería de otra realidad. A diferencia del capítulo 10, aquí solo hay un Rick y Morty, ya que las realidades alternativas planteadas se encuentran jerarquizadas y dependientes de la realidad 1 (R1).

Toda la anterior reflexión, aunque proviene de un dibujo animado, permite comprender la cuestión de las totalidades alternativas, diferenciándolas entre aquellas que resultan paralelas de las que son derivadas.

Si llegáramos a suponer que lo anteriormente planteado es posible, cuestión que algunos astrofísicos sostienen al hablar de infinitos alternativos, estaríamos frente a una explicación de niveles de realidad, es decir, de jerarquizaciones de realidades derivadas o de realidades distintas o alternativas (Mayo, 2016). Bajo el primer enfoque, los sucesos de R1 se encuentran priorizados jerárquicamente ante lo que suceda en R2, mientras que, en la segunda visión, los sucesos de ambas realidades son igualmente importantes.

Ese argumento, el de realidades dentro de realidades, aparece a menudo en filmes y novelas distópicas como Piso 13 de Josef Ruznak, en el que se presenta una realidad creada y usada como medio de entretenimiento de un hombre casado. Por otro lado, se tienen filmes como Matrix, Nirvana y Avalon que son ejemplos paradigmáticos del juego de las realidades múltiples (Herrero, 2015).

Este tipo de explicaciones también se pueden considerar cuando se habla de la realidad virtual, ya que si se toma en cuenta que las "realidades otras" son cosas que parecen reales pero no lo son, entonces la realidad virtual pertenece a este ámbito, pudiendo cuestionar si acaso lo que ahí se hace puede o no ser juzgado como si se hiciera "realmente" (Leiva, 2013).

$\mathrm{Al}$ enfocarse en el tema de las series televisivas, no pueden quedar fuera los análisis que se han realizado sobre la telerrealidad, concepto que cuestionó la noción de realidad que se visualizaba en las pantallas a finales del siglo XX. La telerralidad dio pauta, entre muchas otras cuestiones de índole mediático, a un fenómeno de entretenimiento y morbo de la audiencia, la cual deseaban estar al tanto de lo que "realmente pasaba" en estos programas (Chicharro, 2009). Telerrealidades como 
Big Brother, llevaron a pensar que lo que se veía en las pantallas era "la realidad", aunque también abrían la pauta a cuestionar, si lo que se transmitía era "una realidad" creada simplemente para el entretenimiento de los televidentes (Mateos, 2011).

A diferencia de la televisión fantástica o ficticia, la telerrealidad busca recrear o presentar situaciones "reales", teniendo como medio primordial, el contar con personas "reales" y no con actores, quienes suelen representar personajes creados (Rivera, 2005). Esto último es algo que se ha cuestionado, ya que en múltiples ocasiones, las decisiones, elecciones y comportamiento de los individuos de estas realidades no corresponden a su personalidad fuera de la telerrealidad, por lo que se presume que su conducta llega a ser motivada por las situaciones en las que se les coloca de manera artificial (Cáceres, 2010).

Lo anterior podría ser más simple en el caso de las series de ficción, ya que éstas, aunque coinciden en algunos aspectos con la vida real, se presentan de una manera creativa, incluyendo elementos de fantasía o imaginación provenientes del productor del contenido expuesto (Pardo, 2000). Sin embargo, ¿el hecho que no se presenten o "vendan" como algo real, hace más sencillo o permisible el hacer juicios desde nuestra realidad?, ¿Acaso el que un personaje cometa un delito, golpee traficantes, tenga relaciones amorosas extramaritales o acose a una persona en alguna de estas realidades, no es juzgable por no ser "la realidad"?, y en cuanto a la enseñanza de la ética, ¿Será adecuado o posible hacer juicios éticos de los comportamientos que se realicen en estas realidades alternativas, sean paralelas o derivadas?

\section{Metodología}

\section{Caso de estudio}

Black Mirror es una serie de ficción de nacionalidad británica producida por Endemol, compañía productora de televisión famosa por sus programas de telerrealidad, entre los que se encuentra Big Brother. La serie, en general, busca exponer la manera en que las tecnologías de la información pueden influir en la vida de las personas, desencadenando serios dilemas en y entre sus personajes. Este programa ha sido reconocido con el Premio Emmy a la mejor miniserie o película para televisión en el 2012, contando actualmente con 3 temporadas al aire, aunque se tiene una cuarta en proceso de producción (País, 2016).

$\mathrm{Su}$ controversial nombre, hace referencia a las pantallas que rodean a los individuos en todo momento dentro del mundo contemporáneo, las cuales van desde la televisión, hasta el computador y los equipos móviles personales (Filmaffinity, 2017). Aunque es un programa de ficción, Black Mirror recrea una realidad alterna de tipo futurista, en el que los personajes no son muy distintos de los televidentes, salvo que su entorno se encuentra determinado e influido por la tecnología y los medios de comunicación, algo no muy alejado a nuestra realidad (Rotten Tomatoes, 2014).

Para el presente estudio, se ha realizado una actividad colaborativa con un grupo de estudiantes de pregrado de diferentes áreas profesionales de una institución educativa privada del municipio de Zapopán, México. La población se integró de 30 estudiantes que 
participaron en el curso-taller denominado "Taller de Reconocimiento de problemas éticos contemporáneos. Una aproximación desde Black Mirror" llevado a cabo durante la semana del 25 al 29 de septiembre del 2017.

El objetivo de esta actividad era desarrollar en los participantes la competencia ética de juicio moral, consiguiendo apreciar en las decisiones de los personajes de la serie, la dignidad, necesidades, vulnerabilidades y derechos propios y ajenos, que garantizaran un trato justo entre las personas. Sin embargo, aunque este era el cometido primordial de la semana, toda la actividad se gestó dentro del marco de una pregunta detonante: ¿Podemos juzgar la moralidad de una realidad alternativa desde "nuestra" ética?
El último día del taller, los participantes vieron el capítulo Blanca Navidad, el cual es un episodio especial con el que cierra la segunda temporada de la serie. En éste, los personajes forman parte de varios sucesos que pueden ser considerados dilemáticos, y que se esperaba que los alumnos pudieran identificar y reflexionar, así como hacer un juicio al respecto (Rando, 2014).

De manera previa a la actividad final, los participantes tuvieron una charla acerca de las realidades alternativas, buscando ampliar la reflexión lo más posible. La tabla 1, nos presenta los problemas o dilemas éticos que se identificaron como parte de la actividad:

Tabla n. ${ }^{\circ} \mathbf{1}$

Problemas o dilemas éticos identificados

\begin{tabular}{|l|l|}
\hline Dilema & Situación \\
\hline Tortura & $\begin{array}{l}\text { El manejo de las cookies como entes sin derechos, por no ser la persona, } \\
\text { sino solo una copia de la misma, permite que éstos sean torturados o incluso } \\
\text { enloquecidos con el fin de poder doblegar su voluntad. }\end{array}$ \\
\hline Esclavitud & $\begin{array}{l}\text { Las cookies son creadas con el fin de ser asistentes personales, a lo que no pueden } \\
\text { negarse, ya que son "prisioneros" sin decisión sobre su futuro, el cual de una } \\
\text { forma indirecta, no existe. }\end{array}$ \\
\hline Inteligencia artificial & $\begin{array}{l}\text { ¿Hasta dónde las cookies son copias estáticas del individuo, o cuentan con la } \\
\text { posibilidad de evolucionar en conocimientos, reacciones o incluso emociones?, } \\
\text { ¿Se les puede considerar inteligencia artificial? }\end{array}$ \\
\hline Segregación & $\begin{array}{l}\text { La posibilidad de bloquear a las personas para que simplemente sean segregadas } \\
\text { del espacio vital de las demás personas. Esto podría generar situaciones de } \\
\text { discriminación y desigualdad. }\end{array}$ \\
\hline Violentación de derechos de detenidos & $\begin{array}{l}\text { Al personaje central le es extraída una confesión en contra de su voluntad, lo } \\
\text { que podría cuestionarse si se llega a considerar a su cookie como una extensión } \\
\text { de él mismo. Esto último podría poner en entredicho los argumentos que } \\
\text { respaldan la posibilidad de esclavitud y tortura. }\end{array}$ \\
\hline
\end{tabular}

Fuente: Creación propia. 
Aunque los participantes pudieron identificar diversos problemas o dilemas que se desarrollan durante el capítulo, en todo momento quedaba la duda de si esto era juzgado negativamente solo desde nuestro punto de vista moral, o si sería igual en el caso de que dicha realidad fuera nuestra totalidad. Por ende, y como parte de la reflexión del episodio, se les recordó la pregunta marco; ¿Es posible hacer juicios éticos en acciones realizadas dentro de una realidad alternativa? Las principales respuestas se analizan a continuación.

\section{Análisis de resultados}

De manera general, las respuestas en torno a la posibilidad de juicios éticos en realidades alternas se centraron en 3 hipótesis de análisis:

\section{Hipótesis 1}

Si las realidades alternas son paralelas, cada una de ellas puede visualizarse como una totalidad en sí misma (R1-R1-R1...), y, por ende, siguiendo el principio de que en el mundo cuántico la racionalidad puede cuestionarse, no se puede hablar de una ética universal. Bajo la premisa de que Black Mirror presente una realidad alternativa paralela, la ética se convierte en una moral que no impacta a otras realidades salvo la propia (Figura n. ${ }^{\circ} 1$ ).

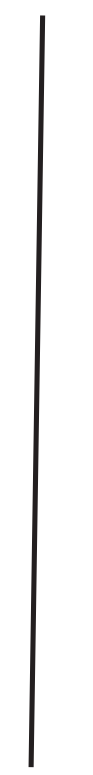

R1

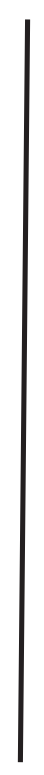

R1

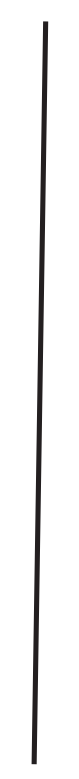

R1

R1
R1

Figura $n .^{\circ}$ 1. Realidades alternativas paralelas. Fuente: Creación propia 


\section{Hipótesis 2}

Si a diferencia de la hipótesis 1 las realidades alternas son jerarquizadas, es decir, que se encuentra una dentro de otra, se puede hablar de realidades derivadas (R1-R2-R3...). Bajo esta idea, las realidades deben apegarse a los principios que regulan a la primera de la que derivan, ya que tienen una dependencia directa o indirecta con ésta. Black Mirror, por ser una serie de televisión creada en la realidad, se deriva y relaciona con los principios y valores de la $\mathrm{R} 1$, pudiendo juzgarse desde la ética como totalidad de esta realidad, así como de todas sus derivadas (Figura n. ${ }^{\circ}$ ).

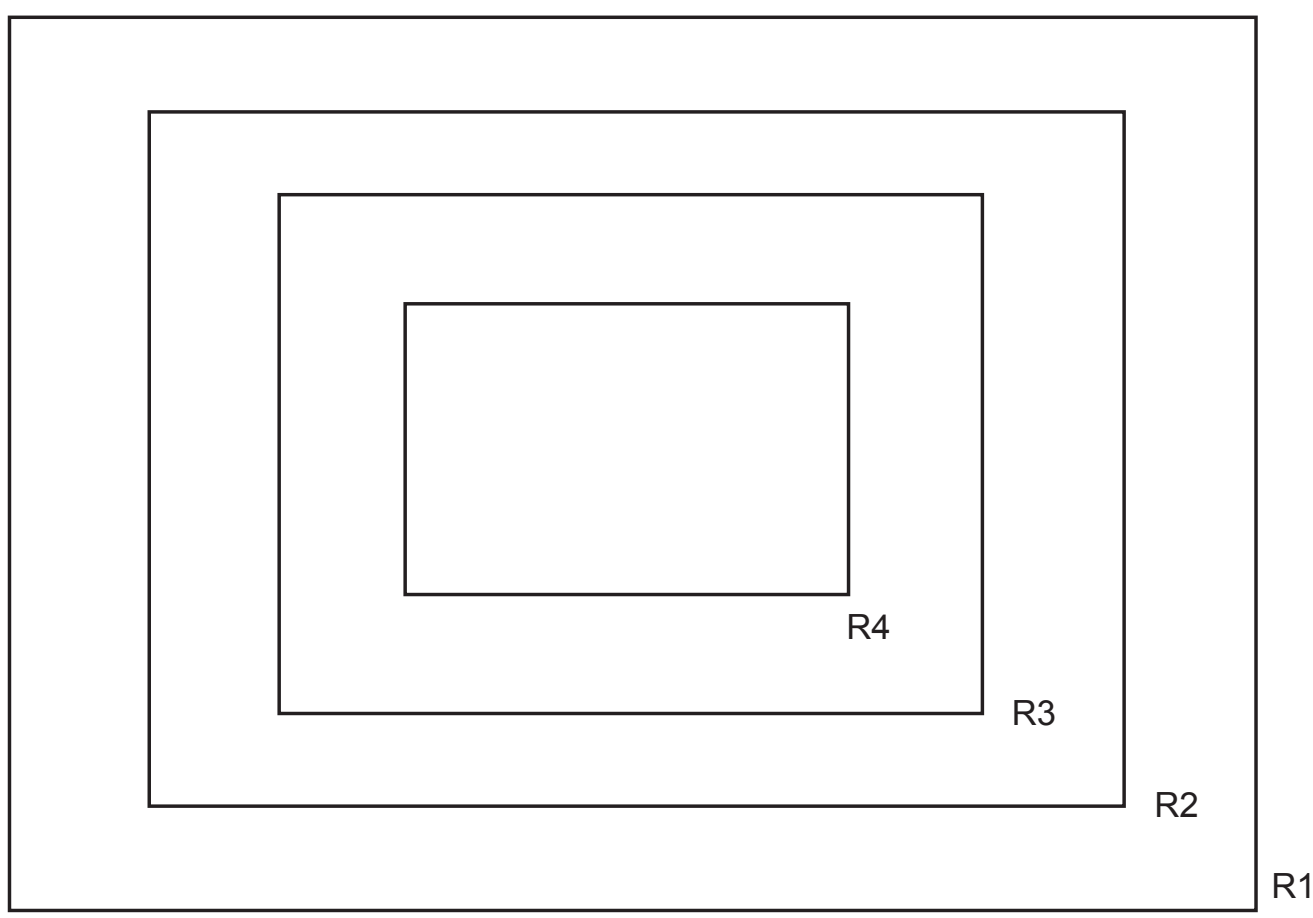

Figura $n .^{\circ}$ 2. Realidades alternativas jerarquizadas o derivadas. Fuente: Creación propia.

\section{Hipótesis 3}

Como variación de la hipótesis 1 , puede darse la situación que las realidades alternativas, aunque sean paralelas, compartan principios fundamentales cuando las mismas se interconectan de alguna forma. Tómese como ejemplo el principio de los agujeros de gusano que suelen usar los astrofísicos. Si las realidades alternativas paralelas se encuentran relacionadas por un agujero de gusano, la totalidad puede ya no implicar una sola realidad, sino el bloque de realidades interconectadas. Bajo esta permisa, si los medios de comunicación permitieran que las realidades alternativas paralelas compartieran espacios en los que se visualizaran unas a otras, 
todas sus realidades, principios y valores se conjugarían en una sola gran R1, la cual sería una totalidad con una ética nuevamente universal (Figura 3).

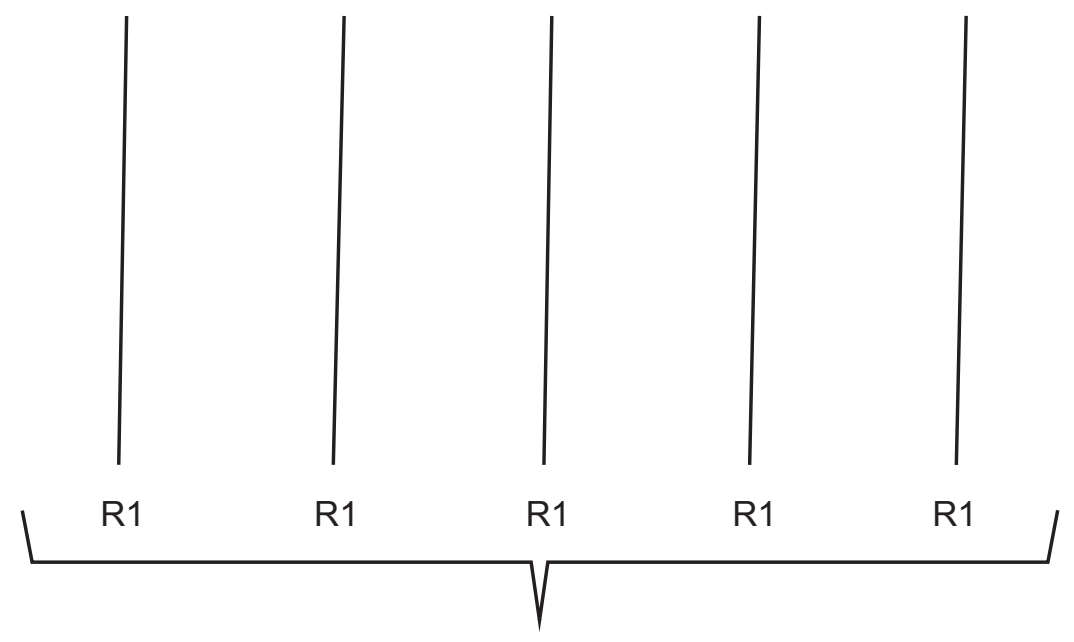

R1

Figura n. ${ }^{\circ}$. Realidades alternativas paralelas interconectadas. Fuente: Creación propia.

Aunque el objetivo central del ejercicio académico parecía centrarse en el desarrollo de la competencia ética en los participantes del taller, las conclusiones alcanzadas resultaron ser mucho más valiosas, ya que les permitió hacer consciente la posibilidad de cuestionar el universalismo de la ética cuando se habla de totalidades alternativas.

La mayoría de los participantes, pudo apreciar que los juicios son acciones que no se pueden dar a la ligera, y que existen situaciones que inevitablemente recaen en casos de inconmensurabilidad. Dar opiniones acerca de otras realidades, espacios o situaciones, careciendo de argumentos sólidos, puede llegar a ser tan riesgoso como la de confiar en correlaciones espurias para tomar decisiones, las cuales más que dar explicaciones objetivas y concretas, pueden resultar ser inciertas, subjetivas y erróneas, aunque psicológicamente persuasivas.

El presente ejercicio académico, buscaba desarrollar la competencia de juicio ético en los participantes, aunque de manera indirecta pretendía dar luz acerca de la posibilidad o imposibilidad de realizar este tipo de juicios sobre realidades propias $y$ alternativas. Al final de cuentas, y viendo las hipótesis alcanzadas, el objetivo del taller se ha conseguido de manera óptima.

\section{Conclusiones}

No cabe duda que lo que este texto presenta podría resultar conflictivo para muchos filósofos o académicos, quienes suelen la objetividad y la certeza sobre el mundo basándose en ideales 
conceptuales, sin embargo, para aquellos que cuestionamos la posibilidad de certeza en un mundo en que la racionalidad plena es una utopía, la idea de las realidades alternativas no resulta ser algo que no pueda considerarse, aunque sea como punto de análisis en ejercicios académicos.

De manera tradicional, la idea del mundo remite a la idea de totalidad, tomando como marco de referencia la totalidad de objetos que plantea la física, y que tiende a relacionarse con objetos espacio temporales que terminan siendo hechos (Tomás, 2006). Sin embargo, la idea de una realidad como totalidad de hechos, implicaría la necesidad de una totalidad que se contuviera a sí misma, lo que según Gabriel (2016), resulta lógicamente imposible. Para este autor, el mundo no existe, y éste es solo la idea de un orden que necesitamos para protegernos del miedo a la infinitud de las cosas (Gabriel, 2016). De esta forma, la realidad es vista únicamente como un contexto que tiene su propio ámbito de objetos (Gabriel, 2015).

Sin embargo, esto mismo puede cuestionarse cuando se habla de valores, ya que al no estar estos en el universo de la física, no deberían de resultar ser un problema, aunque los agentes con los que se relacionan sí se encuentran determinados por la realidad (Gabriel, 2010). Bajo este enfoque, si una persona viajara a realidades alternativas, aunque su contexto cambiara, no se modifica el hecho de que sus actuaciones puedan violentar o respetar sus valores, independientemente de la realidad en la que se encuentra (Hipótesis 3). Por otro lado, para aquellos que se encuentran en dicha realidad, posiblemente la situación sea diferente, ya que dependen de los valores o principios que originalmente tienen (Hipótesis 1). Lógicamente esto no es algo que pueda cuestionarse con las realidades alternativas derivadas o jerarquizadas, ya que éstas dependen de una gran totalidad con su propio contexto (Hipótesis 2).

Se quiere señalar que, planteamientos como los que aquí se presentan, corresponden a diversas problemáticas filosóficas presentes en la relación entre la ética y las realidades alternativas, lo cual es común en la literatura distópica y heterotópica. Estas premisas son usuales en los relatos y novelas de Philip K. Dick, que de manera reciente se ha venido llamando "Nuevo realismo filosófico" y que engloban perspectivas ontológicas de Graham Harman (Harman, 2011) y Markus Gabriel.

A manera de conclusión, se señala que gran parte de estos dilemas filosóficos, sobre la posibilidad de una totalidad en una existencia de realidades alternativas, se centra en la problemática que acontece a partir de la noción que se tiene de modernidad sobre la totalidad del universalismo, lo cual viene a fracturarse bajo enfoques como los que propone Bauman (1996) o Lyotard (1991). La cuestión más complicada, es la de tratar de hacerle ver a alguien, o en este caso a un grupo de alumnos, la posibilidad de cuestionar el universalismo en el que se deposita la ética como una totalidad, lo cual resulta poco sostenible bajo este tipo de propuestas de realidades alternas. En todo caso, los agentes morales deciden con base en sus creencias de tipo normativo, y la aparición de nuevos espacios no debería hacerlos reconsiderar sus creencias valorativas, ya que, en origen, su actuar debería tratar de aplicar el conocimiento valorativo en el uso de estos "nuevos objetos" y no depender únicamente del universalismo o totalidad de lo que consideran correcto. 
Ya sea que se aprecien realidades alternativas ficticias, como se dio con el ejercicio con Black Mirror, o que llegáramos a tener la posibilidad de trasladarnos hacia otras realidades, como lo propondría el dibujo animado de Rick y Morty, esto no debería cuestionar lo que se considera como correcto o incorrecto, ya que aun cuando hubiesen "realidades múltiples"o "realidades otras", los agentes morales no deben de reconsiderar las normas que ya suscriben, las cuales son válidas por sí mismas, y no por su pertenencia a una totalidad ética universal.

El ser humano debe esforzarse por cambiar hacia lo mejor que uno puede ser (desde cualquier perspectiva ética), independientemente de la posibilidad de distintas realidades.

\section{Bibliografía}

Bauman, Z. (1996). Teoría sociológica de la posmodernidad. Espiral, II (5), pp. 81-102.

Cáceres, M. (2010). EL discurso de la televisión en la cultura del espectáculo: los procesos de mediación en los programas de la telerrealidad. Sphera Pública(10), pp. 207-222.

Carrillo, M. E. (2005). Enseñar a ver televisión. Comunicar(25).

Chicharro, M. d. (2009). Información, ficción, telerrealidad y telenovelas: algunas lecturas televisivas sobre la sociedad española y su historia. Comunicación y Sociedad(11), pp. 73-98.

Evans, T. (2015). Wubba Lubba Dub Dub!: The Pursuir of Happiness in Rick And Morty. Under Construction @Keele, pp. 10-17.
Fernandez, R. (Mayo de 2014). Usos y aplicaciones didacticas del cine y la televisión en el aula. Universidad de Castilla-La Mancha. Recuperado de: http://blog.uclm.es/ ricardofdez/2014/05/28/usos-y-aplicacionesdidacticas-del-cine-y-la-television-en-el-aula/

Filmaffinity. (Agosto de 2017). Black Mirror. Recuperado de: https://www.filmaffinity. $\mathrm{com} / \mathrm{mx} /$ film800295.html

Gabriel, M. (2010). ¿Contingencia o necesidad? Schelling y Hegel acerca del estatus modal del espacio lógico. Ideas y valores, 59(142), pp. 5-23.

Gabriel,M. (2015). ¿Por qué no existe el mundo? En M. T. Ramírez, El nuevo realismo. La filosofía del siglo XXI. Morelia: Siglo XXI Editores- UMSNH.

Gabriel, M. (2016). Por qué el mundo no existe. México: Océano.

Harman, G. (2010). Towards Speculative Realism: Essays and Lectures. Ontario: Zero Books.

Harman, G. (2011). The Quadruple Object. Ontario: Zero Books.

Herrero, M. (2015). Realidades alternativas y mundos posibles. La tecnología y los media como vías de acceso a la imaginación. $L a$ trama de la comunicación, 19, pp. 251-262.

Jiménez, E.y Marcos, N. (27 de octubre de 2016). Black Mirror: Todos los episodios ordenados de peor a mejor. El País. Recuperado de: https://elpais.com/cultura/2016/10/26/ television/1477475621_736580.html 
Leiva, R. (2013). La realidad a prueba de lo virtual. Universitas, Revista de CIencias Sociales y Humanas(19), pp. 147-180.

Lyotard,J.-F. (1991). La condición postmoderna. Informa sobre el saber. Madrid: Cátedra.

Mateos, J. (2011). La telerrealidad en las televisiones españolas. Comunicación $y$ Sociedad(15), pp. 169-194.

Mayo, D. (2016). Especulaciones y controversias en la cosmología física contemporánea: el problema de la cocreación en la hipótesis del multiverso, un análisis historico. Revista Colombiana de Filosofía de la Ciencia, 16(33), pp. 213-250.

Observatorio del Ocio y Entretenimiento Digital. (Marzo de 2012). La importancia del uso del cine como medio educativo para niños. Recuperado de: http://www.ocendi.com/ educamedia/la-importancia-del-uso-delcine-como-medio-educativo-para-ninos/

Pardo, V. (2000). 100 películas de cine para trabajar la televisión en el aula. Comunicar(14).
Rando, P. (2014). ¿Es el especial Navidad de Black Mirror la más fofa de sus entregas? Vanity Fair. Recuperado de: http://www. revistavanityfair.es/actualidad/television/ articulos/es-el-especial-navidad-de-blackmirror-jon-hamm-la-mas-fofa-de-susentregas/20117.

Rivera, J. (2005). La ficción y otras realidades. Razón y Palabra, 10(44).

Rotten Tomatoes. (2014). Black Mirror: Season 3. Obtenido de https://www.rottentomatoes. com/tv/black_mirror/s03/

Salcedo, J. (2013). Nibilism and Escapism in Rick and Morty. Curso monográfico de literatura y cultura anglo-americana. Recuperado de: https://www.academia.edu/29293324/_ Nihilism_and_Escapism_in_Rick_and_ Morty_2013_

Tomás, R. (2006). La teoría del multiverso y la prueba ontológica de Dios. Invenio, 9(16), pp. 5-8. 